\title{
Reaction time to tones in tonal backgrounds and a comparison of reaction time to signal onset and offset
}

\author{
DAVID S. EMMERICH, LESLIE J. PITCHFORD, and CURTIS A. BECKER \\ State University of New York at Stony Brook, Stony Brook, New York 11790
}

\begin{abstract}
In order to learn more about the processing of auditory information, simple reaction time (RT) measurements were made when subjects were asked to respond to tones presented against a tonal background. In the first experiment, a counterintuitive result was obtained: with two widely separated frequencies, 1,900 and $4,000 \mathrm{~Hz}$, the presence of the background facilitated RT. In a second experiment, using two frequencies which were closer together $(3,500$ and $4,000 \mathrm{~Hz})$, slowing of RT in the presence of the background was observed, as would be expected from the literature on auditory masking. A third experiment suggested that the information carried by signal offset is not an important factor in the facilitation observed with widely separated tones. In this experiment an unexpected result was found: subjects receiving offsets in their right ears responded significantly more slowly than did subjects receiving offsets in their left ears.
\end{abstract}

A number of studies have investigated various aspects of the human observer's response to combinations of sinusoidal stimuli. As well as having practical value, such studies have yielded much basic information about auditory processing. Among the diverse topics which have been investigated are the masking of one tone by another, as studied by Wegel and Lane (1924) and numerous subsequent researchers, and the perceived loudness of tonal complexes, as studied by Fletcher and Munson (1933) and many others. In addition, beats and combination tones have received much attention in the literature. However, relatively few studies have been addressed to the measurement of simple reaction time (RT) to combinations of sinusoidal stimuli, despite the advantages of simple $R T$ in investigations of auditory information processing. In addition to being a very sensitive index of performance, simple $R T$ is an appealing measure in that the response makes relatively small demands on the judgmental capacities of the subject, and hence the response should be relatively closely related to the underlying neural processing carried out by the auditory system.

Chocholle and associates (Chocholle, 1972; Chocholle \& Greenbaum, 1965, 1966; Chocholle \& Saulnier, 1969) have conducted a number of experiments in which simple RT to a $1,000-\mathrm{Hz}$ signal tone presented monaurally against a background of one or two additional sinusoids was measured. In these studies it was reported that when the back-

Requests for reprints should be sent to David S. Emmerich, Department of Psychology, State University of New York, Stony Brook, New York 11794. The current address of Curtis A. Becker is: Department of Psychology, University of Oregon, Eugene, Oregon 97403. ground was considerably more intense than the signal tone, RT to the signal tone was increased. This effect diminished as the intensity of the signal tone approached that of the background. In fact, from the data presented, it appears that in some conditions when the signal tone is at least as intense as the background the presence of the background may even facilitate RT slightly. Analogous effects were reported for loudness matches.

In the studies of Chocholle and associates, RT data obtained with several backgrounds differing in frequency composition and intensity were presented; the signal frequency was always $1,000 \mathrm{~Hz}$, however. The present study was undertaken with the goals of extending this research by investigating other signal frequencies and of trying to gain further insights into the mechanisms involved.

\section{EXPERIMENT I}

In this experiment, two rather widely separated frequencies, 1,900 and $4,000 \mathrm{~Hz}$, were employed. In order to determine if effects were symmetric with respect to frequency, each of the two frequencies served in turn as a signal and as a background for the other signal frequency.

\section{Method}

Subjects. Twelve undergraduate volunteers from introductory psychology classes were used as subjects. Six of the subjects were female and six were male. All had clinically normal hearing at the frequencies used in the experiment, and none had previously participated in an auditory experiment.

Apparatus. Tonal stimuli were produced by General Radio 1310A oscillators, and noise was produced by a Grason-Stadler 455C noise generator. These stimuli were presented monaurally by means of a matched pair of Telephonics TDH-39 earphones. 
The presentation of signal tones was controlled by means of an electronic switch and interval timer. Subjects were seated in a sound-attenuating chamber.

Procedure. Haif of the subjects received signal tones in the left ear and half received them in the right ear. Each trial began with the presentation of a warning signal consisting of a $200-\mathrm{msec}$ burst of white noise. This noise burst was presented to the nonsignal ear at a spectral level, $\mathrm{N}_{\mathrm{o}}$, of $10 \mathrm{~dB}$ re $0.0002 \mathrm{dynes} / \mathrm{cm}^{2}$. Immediately after the termination of the noise burst, a background tone was presented to the signal ear, and it remained present for the remainder of the trial. The intensity of the background tone took one of four possible values, designated OFF, LOW, MEDIUM, and HIGH, on a given trial. Following a variable foreperiod of $2,21 / 4$, or $2 \frac{1 / 2}{\mathrm{sec}}$, the signal tone was added to this background. The signal tone had a duration of $50 \mathrm{msec}$ and was switched with a $2.5-\mathrm{msec}$ rise/fall time. The intensity of the background tone and the foreperiod duration were selected randomly without restriction for each trial. The subject was instructed to press a telegraph key as quickly as possible after the onset of the signal tone. The telegraph key was mounted on a "right-handed" writing chair, and subjects were instructed to keep one of their right fingers on the key poised to make a response. RT was measured to the nearest millisecond from the onset of the signal tone. RTs shorter than $100 \mathrm{msec}$ or longer than $500 \mathrm{msec}$ were excluded from the data analysis. (Such occurrences were infrequent: less than $3 \%$ of the data were excluded in this fashion.) Immediately after a subject's response, a 2-sec intertrial interval began.

The subjects participated in four 1-h experimental sessions. During the first session, the subjects were given hearing tests, instructed on the task, and given four 35-trial practice blocks. In each of the remaining three sessions, the subjects received eight 35-trial blocks. The first 5 trials of each block were excluded from the data analysis. Subjects were given a short rest and told the sum of their RTs at the end of each block. After the initial practice session, the subjects were informed that the subject whose mean RT improved the most in the remaining three sessions would receive a prize of $\$ 10$.

The subjects were run in pairs, with one subject resting outside the sound-attenuating chamber while data were being obtained from the other subject. The subjects changed places after each two blocks until each subject had completed 8 blocks of trials. In order to control for fatigue and practice effects which might affect comparisons of results obtained with the 1,900 and $4,000-\mathrm{Hz}$ signal tones, stimulus presentations were counterbalanced.

The $1,900-\mathrm{Hz}$ signal tone was presented at a sound pressure level (SPL) of $45 \mathrm{~dB}$ re 0.0002 dynes $/ \mathrm{cm}^{2}$. The $1,900-\mathrm{Hz}$ background tone was either OFF, or at 25,45 , or $65 \mathrm{~dB}$ re 0.0002 dynes $/ \mathrm{cm}^{2}$. The $4,000-\mathrm{Hz}$ signal tone was presented at a sound pressure level of $50 \mathrm{~dB}$ re 0.0002 dynes $/ \mathrm{cm}^{2}$. The $4,000-\mathrm{Hz}$ background tone was either OFF or at 30,50 or $70 \mathrm{~dB}$ re 0.0002 dynes $/ \mathrm{cm}^{2}$. Thus the loudnesses of the corresponding 1,900 - and $4,000-\mathrm{Hz}$ stimuli were approximately equal (Fletcher \& Munson, 1933).

\section{Results and Discussion}

An analysis of variance indicated that the mean RTs obtained from subjects receiving signal tones in the left ear did not differ significantly from those obtained from subjects receiving signal tones in the right ear. For this reason, the data from left-ear and right-ear subjects are combined when presented below. The analysis also indicated that RT to $4,000-\mathrm{Hz}$ tones presented against a $1,900-\mathrm{Hz}$ background was significantly faster than RT to $1,900-\mathrm{Hz}$ tones presented against a $4,000-\mathrm{Hz}$ background $[F(1,10)=7.63, p<.05]$. In addition, RT was found to vary significantly with the intensity of the back- ground tones $[F(3,30)=3.33, p<.05]$. The magnitude of the latter effect appears to be about the same regardless of which tone was the signal: no asymmetry was evident. None of the interactions among the different factors in the experiment were statistically significant.

The mean RTs for the different combinations of signal tone and background intensity are presented in Figure 1. Each mean is based on more than 1,000 observations. The finding that RT is slightly shorter for the $4,000-\mathrm{Hz}$ signal tones than for the $1,900-\mathrm{Hz}$ signal tones was not entirely unexpected. Physiological evidence obtained from animal subjects (e.g., Kiang, 1965) indicates that the latency of onset of activity in auditory nerve fibers is greater the greater the distance from the stapes to the place of origin of the fibers on the basilar membrane. This distance is shorter for fibers tuned to higher frequencies, and thus neural activity should be available slightly faster from those fibers most responsive to the higher frequency tone. The magnitude of the difference in RT to the two signal frequencies is somewhat greater than would be expected on this basis alone, however: more central processes are presumably involved as well.

The finding that $R T$ varies significantly with intensity was also not unexpected, but the direction of the effect shown in Figure 1 is rather surprising. Instead of increasing with increasing background intensity (as would have been expected both from the studies of Chocholle and his associates cited above and from masking experiments), RT decreases. Two questions immediately present themselves. What mechanisms could account for a decrease in

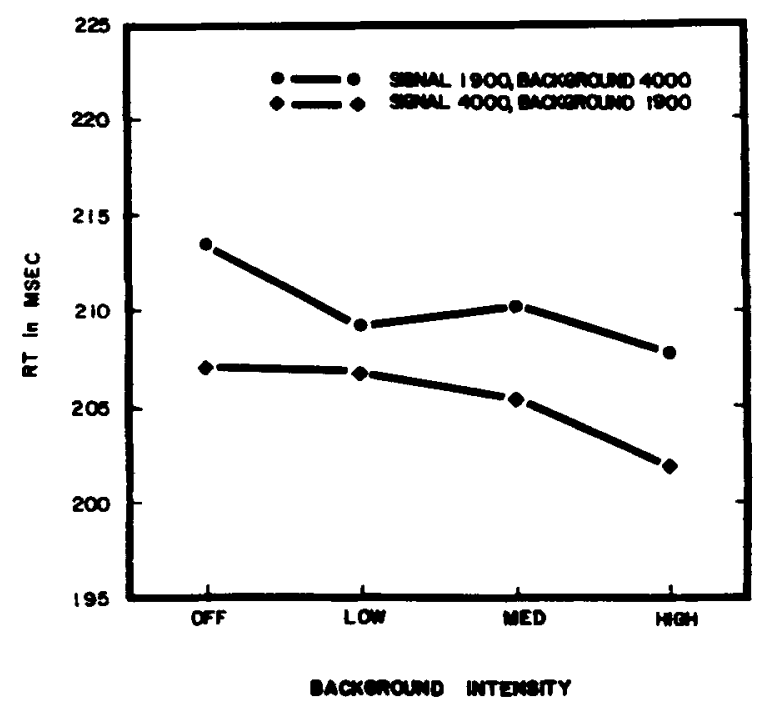

Figure 1. Mean reaction time to signal tones presented against a tonal background. The 1,900-Hz signal (circles) had an SPL of $45 \mathrm{~dB}$, and the 4,000-Hz signal (diamonds) had an SPL of $50 \mathrm{~dB}$. The 1,900-Hz background was either off or at an SPL of 25 , 45 , or $65 \mathrm{~dB}$. The $4,000-\mathrm{Hz}$ background was either off or at an SPL of 30, 50, or $70 \mathrm{~dB}$. 
RT as the background intensity increases, and what could account for the difference between the present findings and the increase in RT found in similar experiments by Chocholle and Greenbaum (1965, 1966)?

A consideration of the results of physiological experiments suggests a possible basis for the decrease in RT to the signal which was observed in the present experiment. A number of researchers (Kiang, 1965; Nomoto, Suga, \& Katsuki, 1964; and many others) have examined the responses of single auditory nerve fibers to combinations of sinusoidal stimuli, and have observed what is known as two-tone inhibition. In studies of two-tone inhibition, it is found that the ongoing rate of discharge of an auditory nerve fiber responding to a tone at the frequency to which it is most sensitive is slowed when a second tone is presented. A further point of interest is that when the second tone is turned off there is a relatively large transient increase in the discharge rate of the nerve fiber, followed by a return to the previous ongoing rate of discharge. Now, we might expect the responsiveness of the auditory fibers most sensitive to the brief signals of the present experiment to be lessened by the presence of a tonal background (as a consequence of two-tone inhibition). However, the presentation of the brief signals would also be expected to lead to a decrease in the ongoing activity of fibers responding to the tonal background, followed by a transient increase in their activity. Both such a decrease and such a transient increase would, of course, be perfectly good cues on which to base a response. To the extent that these cues outweighed any lessening of response in nerve fibers most responsive to the signal itself, RT would be facilitated. Unfortunately, in addition to being quite speculative, this account is incomplete; the conditions under which such cues would outweigh any lessening of response need to be specified. Such specification is not likely to be a simple task. For example, from the results presented in Figure 1 it might be concluded that the more activity that is present as a result of the background tone, the more that RT to the signal tone will be facilitated. In view of the fact that the (presumably physical) basis of two-tone inhibition is not yet well understood, such a conclusion would be premature. In fact, additional data, presented below, show this conclusion to be false. It should also be pointed out that Chocholle (1972) and Chocholle and da Costa (1971) show that background tones presented to the contralateral ear have a small effect on RT to the signal tone. Such an effect would, of course, argue against peripheral explanations of the results. These authors point out, however, that at the intensities used in their experiments the background tones may have reached the ipsilateral ear by bone conduction. More data, both psychophysical and physiological, are needed in order to clarify the situation.
There are several differences in the procedures employed in the present experiment and in those of Chocholle and Greenbaum $(1965,1966)$ which might account for the differences in the results that were reported. The frequency separation between the signal and background tones was much greater in the present experiment than was the case for the data presented by Chocholle and Greenbaum, and the signal duration in the present experiment was much shorter. In Experiment II, a smaller frequency separation between the signal and background tones was employed.

\section{EXPERIMENT II}

In this experiment, the frequencies employed were 3,500 and $4,000 \mathrm{~Hz}$. The separation between these two frequencies is less than present in Experiment I in which RT was speeded by the presence of the background. The separation is greater, however, than that for which Chocholle and Greenbaum (1966) found a slowing of RT as background intensity was increased.

\section{Method}

Subjects. As in Experiment I, 12 undergraduate volunteers from introductory psychology classes were used as subjects. Four of the subjects were female and eight were male. All had clinically normal hearing at the frequencies used in this experiment and none had previously participated in an auditory experiment.

Apparatus. The apparatus was the same as that used in Experiment I.

Procedure. The procedure was the same as that used in Experiment I, except that the frequencies employed were 3,500 and 4,000 $\mathrm{Hz}$. When either frequency was employed as a signal tone, its SPL was $50 \mathrm{~dB}$ re 0.0002 dynes $/ \mathrm{cm}^{2}$. The background tones of either frequency were either OFF or at 30,50 , or $70 \mathrm{~dB}$ re $0.0002 \mathrm{dynes} / \mathrm{cm}^{2}$.

\section{Results and Discussion}

As in Experiment $I$, an analysis of variance indicated that the mean RTs of left-ear and right-ear subjects did not differ significantly, and thus their data are combined when presented below. In Experiment II, the mean RTs to the more closely spaced signal tones did not differ significantly. There was again a significant effect of the intensity of the background tones on $R T$ to the signal tones $[F(3,30)=$ 16.77, $\mathrm{p}<.001$ ]. As can be seen in Figure 2, however, the nature of the intensity effect differs markedly from that observed in Experiment $I$. There was no evidence of any slowing of RT due to the presence of a background for any of the intensities used in Experiment I. In Experiment II, there is no evidence that the presence of a background speeds RT (the apparent decreases in RT seen in Figure 2 for the LOW and MEDIUM backgrounds are not statistically significant), and the HIGH background slows RT dramatically. The only significant interaction in Experiment II is that between signal frequency and background intensity $[F(3,30)=4.25$, $\mathrm{p}<.05$ ]. As can be seen in Figure 2, although RT 


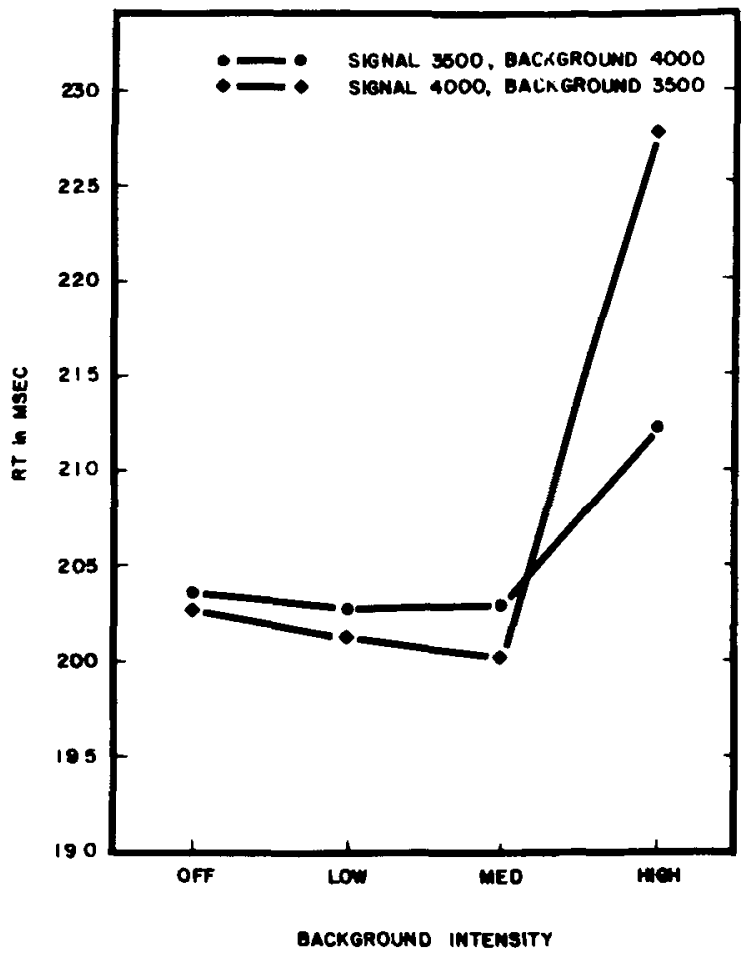

Figure 2. Mean reaction time to signal tones preseated againgt a tonal background. The 3,500-Hz signal (circles) and the 4,000-Hz signal (diamonds) each had an SPL of 50 dB. Background tones were either off or at an SPL of 30,50, or $70 \mathrm{~dB}$.

to the $4,000-\mathrm{Hz}$ tone is slightly faster than that to the 3,500-Hz tone for the OFF, LOW, and MEDIUM background conditions, RT in the HIGH background condition is much slower when the signal frequency is $4,000 \mathrm{~Hz}$ than when it is $3,500 \mathrm{~Hz}$. In other words, the high-intensity $3,500-\mathrm{Hz}$ background tone slows RT to the $4,000-\mathrm{Hz}$ signal tone considerably more than the high-intensity $4,000-\mathrm{Hz}$ background tone slows RT to the $3,500-\mathrm{Hz}$ signal tone. This asymmetry is exactly what would be expected from the literature on masking, in which it is well established that (except at low intensities) low-frequency tones tend to mask high-frequency tones much more readily than high-frequency tones mask low-frequency tones.

The slowing of RT observed in Experiment II makes this experiment more consistent with the data presented by Chocholle and Greenbaum (1966) than is Experiment I. Presumably, if the frequency separation were made even smaller than it was in Experiment II, the slowing of RT would be observed at still lower intensities of the background tone.

In Experiment III, an attempt was made to gain some insight into the basis of the unexpected decrease in RT which was observed in Experiment I when a background tone was present.

\section{EXPERIMENT III}

It was suggested earlier that a factor in the de- crease in RT observed in Experiment I when a background tone was present might have been a modulation of the ongoing rate of activity of neurons responding to the background. The onset of the signal tone would be expected to lead to inhibition of this ongoing activity, and the offset of the signal tone should lead to a transient rebound from such inhibition. One way of investigating the importance of such a transient rebound would be to deprive the subject of signal offsets. This was done in Experiment III by having signals response-terminated rather than of fixed duration. In addition, it was decided to see what the effect would be of giving subjects the task of responding to the offset of a signal which was already present, rather than to the onset of a signal which was initially absent. This task deprives the subject of the information carried by signal onsets. In Experiment III, the signal frequency was always $1,900 \mathrm{~Hz}$, and the background frequency was always $4,000 \mathrm{~Hz}$.

\section{Method}

Subjects. Again 12 undergraduate volunteers from introductory psychology classes were used as subjects. Six of the subjects were female and six were male. All had clinically normal hearing at the frequencies used in this experiment and none had previously participated in an auditory experiment.

Apparatus. The apparatus was the same as that in the preceding two experiments.

Procedure. The procedure employed in this experiment was, in most respects, similar to that employed in the previous two experiments. However, the signal frequency was always $1,900 \mathrm{~Hz}$ and the background frequency was always $4,000 \mathrm{~Hz}$. (The signal and background intensities were the same as those used in Experiment I.) Instead of interchanging the signal and background frequencies, as was done in the earlier experiments, the subject in this experiment was asked to respond to signal ONSET in some blocks of trials and to signal OFFSET in other blocks of trials. With the exception of this difference, the procedure was the same as that used in the previous experiments.

The task in the ONSET condition was like that given to the subject in the previous two experiments, except that after the signal tone came on it remained on until the subject had responded. In the OFFSET condition, the signal tone came on at the beginning of the trial at the same time as did the background tone. After a variable foreperiod, the signal tone went off and the subject pressed the telegraph key as rapidly as possible.

\section{Results and Discussion}

An analysis of variance indicated a marginally significant difference $[F(1,10)=4.54, p<.1]$ in the mean RTs obtained from left-ear and right-ear subjects. The analysis also indicated a significant difference between RT in the ONSET and OFFSET conditions $[F(1,10)=144.02, p<.001]$ and a significant interaction between the ONSET-OFFSET variable and ear of presentation $[F(1,10)=8.15, p<.025]$. As can be seen in Figure 3, RT to signal OFFSET is considerably slower than RT to signal ONSET, and this difference is much greater for right-ear subjects than for left-ear subjects. The finding of a slower RT to OFFSET is consistent with previous research (Goldstone, 1968), but the observation that the 


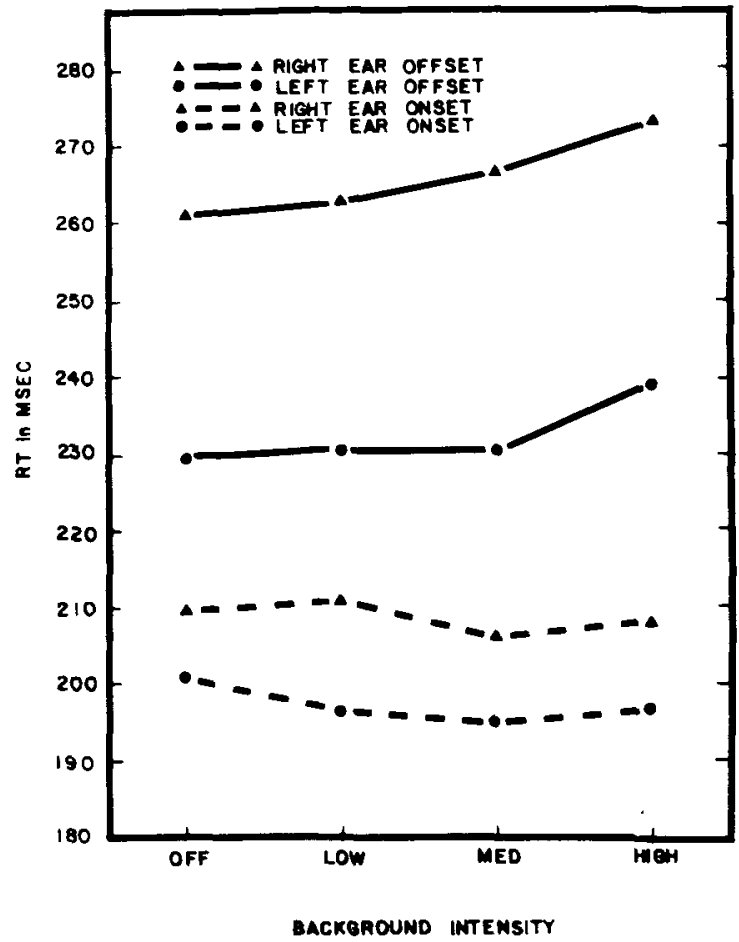

Figure 3. Mean reaction time to onset (dashed lines) and offset (solid lines) of signal tones presented against tonal backgrounds. The signal frequency was $1,900 \mathrm{~Hz}$ and its SPL was $45 \mathrm{~dB}$. The background frequency was $4,000 \mathrm{~Hz}$, and it was either off or at an SPL of 30,50 , or $70 \mathrm{~dB}$. Signals presented to the left ear are represented by circles; those presented to the right ear are represented by triangles.

difference between ONSET and OFFSET RT is much larger for subjects receiving signals in the right ear than for subjects receiving them in the left ear is surprising. This latter finding is attributable to the fact that the right-ear subjects responded significantly more slowly than the left-ear subjects in the OFFSET condition $[\mathrm{t}(10)=2.98, \mathrm{p}<.01]$, while their responses did not differ significantly in the ONSET condition $[\mathrm{t}(10)=1.02, \mathrm{p}>.10]$. The observed results are unlikely to be due to differences between left and right earphones: a pair of earphones which had been matched on the basis of their response characteristics was used, and differences in output of the two phones both at the signal frequency and at the background frequency were less than $1 \mathrm{~dB}$. It is possible that the differences between left-ear and right-ear subjects which were found in the present experiment reflect differences in processing of the sort found, for example, in numerous studies of hemispheric specialization. This matter is currently being examined in more detail in an experiment in which the comparisons between RT for left-ear and right-ear presentations are being made within subjects.

The analysis of variance indicated a significant effect of background intensity on RT $[F(3,30)=3.04$, $\mathrm{p}<.05$ ] as well as an interaction of the effect of intensity with the ONSET-OFFSET manipulation
$[F(3,30)=5.31, p<.005]$. As can be seen in Figure 3, when subjects responded to ONSET the background tended to decrease RT, but when subjects were responding to OFFSET the background tended to increase RT. The decrease of RT in the ONSET condition is of the same order of magnitude as that observed in Experiment I. Furthermore, the mean RT to ONSET is quite comparable to that to the $1,900-\mathrm{Hz}$ signal in Experiment $\mathrm{I}$, while the mean RT to OFFSET is much slower. On this basis, it seems reasonable to conclude that cues provided by signal onset are of primary importance in the facilitation of RT observed in Experiment $I$ and the signal offset is a less important factor. This conclusion should be viewed with some caution for two reasons, however. First, the fact that the signals in the ONSET condition of Experiment III were of longer duration than were the signals in Experiment I would be expected to influence RT somewhat (e.g., see Emmerich, Gruenfeld, \& Wiesenfeld, 1973), and such an effect might interact with the background tone intensity. Second, the fact that the signal tones in the OFFSET condition were on for over $2 \mathrm{sec}$ would be expected to lead to adaptation and consequently to a slowing of RT in this condition.

\section{REFERENCES}

Сносноle, R. Effets de masque homolatéraux et contralatéraux, totaux et partiels, sur un son pur de deux autres sons purs placés de part ed d'autre du premier. Acustica, 1972, 27, 267-277.

Chocholle, R., \& DA Costa, L. Les temps de réaction à un son pur partiellement masqué contralatéralement par deux autres sons purs simultanés choisis de part et d'autre du premier. Comptes Rendus des Séances de la Socièté de Biologie et de Ses Filiales, 1971, 165, 36-41.

Chocholle, R., \& Greenbaum, H. Les temps de réaction aux sons partiellement masqués. Journal de Physiologie, Paris, 1965, 57, 588. (Abstract)

Chocholle, R., \& Greenbaum, H. La sonie de sons purs partiellement masqués. Étude comparatif par une méthode d'eǵalisation et par la méthode des temps de réaction. Journal de Psychologie Normale et Pathologique, 1966, 63, 385-414.

Chocholie, R., \& SAurnier, C. Les temps de réaction à un son partiellement masqué par deux sons simultanés situés de part et d'autre du premier. Joumal de Physiologie, Paris, 1969, 61 . supplément 2, 246. (Abstract)

Emmerich, D. S., Gruenfeld, L. S., \& Wiesenfeld, A. R. Reaction time to noise bursts of different durations. Journal of Experimental Psychology, 1973, 100, 422-423.

Fletcher, H., \& Munson, W. A. Loudness, its definition, measurement and calculation. Journal of the Acoustical Society of America, 1933, 5, 82-108.

Goldstone, $S$. Reaction time to onset and termination of lights and sounds. Perceptual and Motor Skills, 1968, 27, 1023-1029.

KIANG, N. Y. S. Discharge patterns of single fibers in the cat's auditory nerve. Cambridge, Mass: M.I.T. Press, $1 \% 5$.

Nomoтo. M.. Suga, N., \& KATsukI, Y. Discharge pattern and inhibition of primary auditory nerve fibers in the monkey. Journal of Neurophysiology, 1964, 27, 768-787.

WEGEL, R. L., \& LANE, C. E. The auditory masking of one pure tone by another and its probable relation to the dynamics of the inner ear. Physical Review, 1924, 23, 266-285.

(Received for publication March 8, 1976; accepted June $20,1976$. 